\title{
Model Predictive Control Of PWM Rectifier Supplied By Unbalanced Source Voltage
}

\author{
Imad MERZOUK \& Mohamed Lokmane BENDAAS
}

\begin{abstract}
This paper proposes a new control of pulse width modulation PWM rectifier supplied by unbalanced voltage source where model based predictive control (MPC) is the main control method. Using this new control, it is possible to obtain sinusoidal input current with unity power factor under unbalanced conditions. This control requires two steps. First, the unbalanced phase voltage and input line current are decomposed on separate positive and negative sequences. Second, compensated active and reactive powers are calculated in function of the sequences of voltage and current and added to the referencing one. Thus the referencing currents are calculated from the resulting active and reactive powers. Based on discrete model of the PWM rectifier the future value of currents are determined, next the switching state from the eight possible switching states that gives the minimum cost function is selected to be applied on a semiconductor device. The model based predictive control of PWM rectifier with the unbalance voltage source possibility was simulated. Simulation results verified the effectiveness and fast dynamic response of the proposed control strategy.
\end{abstract}

Keywords - model based predictive control (MPC), PWM rectifier, unbalanced voltage supply, active and reactive powers.

\section{Introduction}

Due to the multiple advantages of PWM rectifier such as low line current distortion, high power factor and smooth and controlled dc- voltage source [1], its usage is increasing day by day in several industrial areas and as an input stage of wind turbine conversion system. However, since the PWM rectifier is connected directly to the grid, any unbalance or distortion in the grid causes dysfunctional sequences in the operation of the converter [2-3], hence non desired pulsation terms in the output DC-link voltage are created and the line current becomes unbalanced and distorted. Therefore, the converter loses its main advantages. Researchers are intensively investigating methods to control the PWM converter when the grid is unbalance by improving or modifying the classical existing control strategies and adapting them to remove the effect of unbalance on the operation of the converter. Vector control and direct power control are the most responded strategies to control the PWM rectifier. [3] proposes a modified vector oriented

\section{Imad MERZOUK}

Applied Automation and Industrial Diagnostic Laboratory, Faculty of

Science and Technology, University of Djelfa,

Algeria.

Mohamed Lokmane BENDAAS

Department of Electrical Engineering, Faculty of Technology, University of Batna,

Algeria. control in which the reference current is calculated from the power expressions and two parallel current controllers in positive and negative reference frame one for positive sequence and the second for negative sequence are used. [4] reduce the controllers to one positive reference frame and introduce the concept of resonant controllers to eliminate steady state error because the negative sequence appears with twice grid frequency in positive reference frame. These methods give good results and significantly improve significantly the behavior of PWM rectifier under unbalanced source. But the tuning of Proportional integral (PI) gains and resonant controllers parameters is still a barrier in front of the use of these methods. Thus, researchers focus moved towards another method called direct power control (DPC) as it is more robust than voltage oriented control. in [6] a modified DPC for PWM rectifier was proposed to achieve balanced input current under unbalance voltage operation. Succeed to this idea [7] and [8] added two new control targets: obtaining sinusoidal and symmetrical grid current and removing reactive power ripples or removing active power ripples.

In this paper the same concept of DPC to overcome the effect of unbalance in the performance of PWM rectifier is used, but instead of the hysteresis controllers and lock-up table model predictive control (MPC) proposed in [9-12] is introduced to get directly the switching state that gives the minimum cost function from all possible switching states[11]. Thus, fast time dynamic response is obtained, and the capability of PWM rectifier under voltage unbalance right-through is significantly improved. The performance of MPC under unbalance voltage has been examined by simulations in Matlab /simulink environment.

\section{Control strategy}

The overall control structure consists of two nested regulation loops, the outer dc link voltage regulating loop and the inner ac current regulation loop. The outer voltage loop determines the reference signals for the inner loop according to the objectives such as will be describe in the next section. The inner loop determines the switching state that apply on the of rectifier semiconductor using current Model predictive control (MPC).

\section{A. PWM rectifier modeling}

The model of PWM converter in rotating frame is determined from figure 1 and given by:

$$
\left\{\begin{array}{l}
E_{\alpha}-V_{\alpha}=L \frac{d i_{\alpha}}{d t}+R i_{\alpha} \\
E_{\beta}-V_{\beta}=L \frac{d i_{\alpha}}{d t}+R i_{\beta}
\end{array}\right.
$$




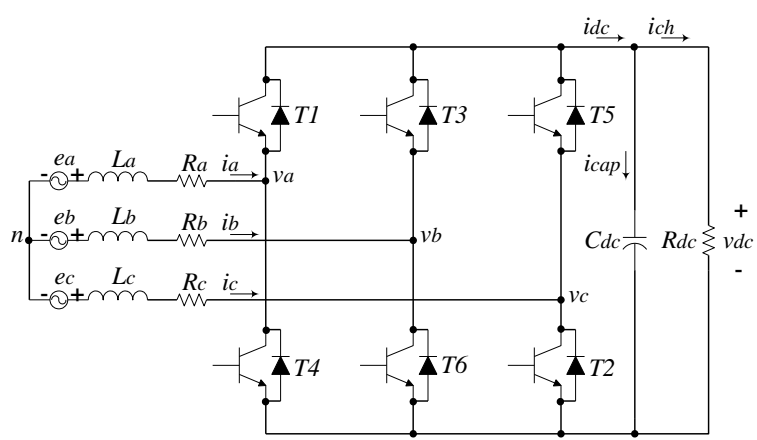

Where:

Figure 1. Structure of the 3-phase PWM rectifier

$e_{\alpha}, e_{\beta}$ : Source voltage in rotating frame.

$V_{\alpha}, V_{\beta}$ : Converter terminals voltage.

$i_{\alpha}, i_{\beta}$ : Line current.

$L, R$ : input filter inductance and resistance.

\section{B. Reference current calculation}

\section{1) Reference current equation}

Using the space vector notation and assuming that the voltage source supply is balanced and harmonic free, the apparent power is given by:

$$
S=P+j Q=\frac{3}{2} E_{\alpha \beta} \times \hat{I}_{\alpha \beta}
$$

Where

$$
E_{\alpha \beta}=E_{\alpha}+j E_{\beta} \quad / I_{\alpha \beta}=I_{\alpha}+j I_{\beta}
$$

$S, P, Q$ : are apparent, active and reactive powers.

After substituting voltage and current by their values shown in (3), we get the active and reactive power shown in (4) and (5) consecutively.

$$
\begin{gathered}
P=\frac{3}{2}\left(E_{\alpha} I_{\alpha}+E_{\beta} I_{\beta}\right) \\
Q=-\frac{3}{2}\left(E_{\alpha} I_{\beta}-E_{\beta} I_{\alpha}\right)
\end{gathered}
$$

The referenced active power determines the DC-link voltage level. And the referenced reactive power is set to 0 to achieve the unity power factor.

$$
\left\{\begin{array}{l}
i_{\text {dref }}=\frac{2}{3 \Delta}\left(E_{\alpha} * P_{\text {ref }}+E_{\beta} * Q_{r e f}\right) \\
i_{\text {qref }}=\frac{2}{3 \Delta}\left(E_{\beta} * P_{\text {ref }}-E_{\alpha} * Q_{r e f}\right)
\end{array}\right.
$$

Where

$\Delta=E_{\alpha}^{2}+E_{\beta}^{2}$. The subscript (ref) means reference value.

\section{2) Unbalance effecte compensation}

The space vector of unbalanced voltage and current is given by[12]:

$$
\begin{gathered}
E_{\alpha \beta}=\left(E_{\alpha}^{+}+E_{\alpha}^{-}\right)+j\left(E_{\beta}^{+}+E_{\beta}^{-}\right) \\
I_{\alpha \beta}=\left(I_{\alpha}^{+}+I_{\alpha}^{-}\right)+j\left(I_{\beta}^{+}+I_{\beta}^{-}\right)
\end{gathered}
$$

Where: superscript $(+,-)$ means positive and negative sequences.

The apparent power is given by (2). After substituting the voltage and current by their values shown in (7) and (8) we get a real part shown in (9) and an imaginary part shown in (10).

$$
\begin{aligned}
& P=\frac{3}{2}\left(E_{\alpha}^{+} I_{\alpha}^{+}+E_{\beta}^{+} I_{\beta}^{+}+E_{\alpha}^{-} I_{\alpha}^{-}+E_{\beta}^{-} I_{\beta}^{-}+E_{\alpha}^{+} I_{\alpha}^{-}+E_{\beta}^{+} I_{\beta}^{-}+\right. \\
& \left.E_{\alpha}^{-} I_{\alpha}^{+}+E_{\beta}^{-} I_{\beta}^{+}\right)
\end{aligned}
$$

$$
\begin{aligned}
& Q=-\frac{3}{2}\left(E_{\alpha}^{+} I_{\beta}^{+}-E_{\beta}^{+} I_{\alpha}^{+}+E_{\alpha}^{-} I_{\beta}^{-}-E_{\beta}^{-} I_{\alpha}^{-}+E_{\alpha}^{+} I_{\beta}^{-}-E_{\beta}^{+} I_{\alpha}^{-}+\right. \\
& \left.E_{\alpha}^{-} I_{\beta}^{+}-E_{\beta}^{-} I_{\alpha}^{+}\right)
\end{aligned}
$$

Compared to active and reactive powers obtained under ideal voltages supply, many additional terms appear under unbalanced voltage supply. These terms result from the interaction between each sequence of the voltage (positive, negative) and the sequences of the current separately. These additional terms are responsible of the poor performance of the rectifier.

According to (9) and (10) the active and reactive powers can be regrouped in two terms:

Where:

$$
P=P_{1}+P_{2}
$$

$$
\begin{aligned}
& P_{1}=\frac{3}{2}\left(E_{\alpha}^{+} I_{\alpha}^{+}+E_{\beta}^{+} I_{\beta}^{+}+E_{\alpha}^{-} I_{\alpha}^{-}+E_{\beta}^{-} I_{\beta}^{-}\right) \\
& P_{2}=\frac{3}{2}\left(E_{\alpha}^{+} I_{\alpha}^{-}+E_{\beta}^{+} I_{\beta}^{-}+E_{\alpha}^{-} I_{\alpha}^{+}+E_{\beta}^{-} I_{\beta}^{+}\right)
\end{aligned}
$$

$\mathrm{P}_{1}$ is the average active power delivered to the DC-link voltage and it is a constant power.

$\mathrm{P}_{2}$ represents the interaction between the positive and negative sequences of the voltages and currents that generates an oscillation in the active power with a frequency that is twice the fundamental frequency.

The same analysis was carried for the reactive power:

$$
\begin{gathered}
Q=Q_{1}+Q_{2} \\
Q_{1}=-\frac{3}{2}\left(E_{\alpha}^{+} I_{\beta}^{+}-E_{\beta}^{+} I_{\alpha}^{+}+E_{\alpha}^{-} I_{\beta}^{-}-E_{\beta}^{-} I_{\alpha}^{-}\right) \\
Q_{2}=-\frac{3}{2}\left(E_{\alpha}^{+} I_{\beta}^{-}-E_{\beta}^{+} I_{\alpha}^{-}+E_{\alpha}^{-} I_{\beta}^{+}-E_{\beta}^{-} I_{\alpha}^{+}\right)
\end{gathered}
$$

There are many control laws that can be applied in the proposed control. However, we chose an important control law that gives balanced and harmonics- free line currents.

Therefore, $I_{\alpha}^{-}=I_{\beta}^{-}=0$, and at the end (9) and (10) can be written as (17) and (18).

$$
\begin{gathered}
P=\frac{3}{2}\left(E_{\alpha}^{+} I_{\alpha}^{+}+E_{\beta}^{+} I_{\beta}^{+}+E_{\alpha}^{-} I_{\alpha}^{+}+E_{\beta}^{-} I_{\beta}^{+}\right) \\
Q=-\frac{3}{2}\left(E_{\alpha}^{+} I_{\beta}^{+}-E_{\beta}^{+} I_{\alpha}^{+}+E_{\alpha}^{-} I_{\beta}^{+}-E_{\beta}^{-} I_{\alpha}^{+}\right)
\end{gathered}
$$

Under the balanced and perfectly sinusoidal grid voltage supply, only the positive sequence component exists, and the powers can be described as:

$$
\begin{gathered}
P=\frac{3}{2}\left(E_{\alpha}^{+} I_{\alpha}^{+}+E_{\beta}^{+} I_{\beta}^{+}\right) \\
Q=-\frac{3}{2}\left(E_{\alpha}^{+} I_{\beta}^{+}-E_{\beta}^{+} I_{\alpha}^{+}\right)
\end{gathered}
$$

It can be seen from (17)-(20) that if we want to eliminate the effect of the negative sequence component, the active and reactive power compensated components can be obtained as:

$$
\begin{aligned}
& P_{\text {comp }}=-\frac{3}{2}\left(E_{\alpha}^{-} I_{\alpha}^{+}+E_{\beta}^{-} I_{\beta}^{+}\right) \\
& Q_{\text {comp }}=\frac{3}{2}\left(E_{\alpha}^{-} I_{\beta}^{+}-E_{\beta}^{-} I_{\alpha}^{+}\right)
\end{aligned}
$$

The modified DPC strategy is based on the idea of injecting the active and reactive power compensated components in the original referenced power to achieve control objectives.

The multiple complex coefficient filter (MCCF) proposed by [13] is used to separate the positive and negative sequences of voltage and current. 
Proc. of The Fifth Intl. Conf. On Advances in Computing, Electronics and Electrical Technology - CEET 2016 Copyright (C) Institute of Research Engineers and Doctors, USA .All rights reserved. ISBN: 978-1-63248-087-3 doi: 10.15224/ 978-1-63248-087-3-14

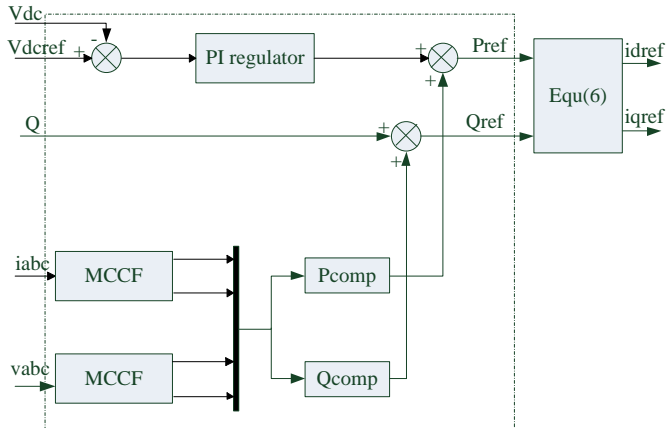

Figure 2. Reference current calculation.

\section{Model predictive control}

In order to estimate the future value of reference current, the discrete time model of the system is needed.

Using Euler approximation [12]:

$$
\frac{d i}{d t}=\frac{i(k+1)-i(k)}{T}
$$

Substituting (23) in (1) we find the discrete model shown in

$$
\begin{gathered}
{\left[\begin{array}{l}
i_{\text {aref }}(k+1) \\
i_{\beta r e f}(k+1)
\end{array}\right]=\left[\begin{array}{cc}
1-\frac{R T}{L} & 0 \\
0 & 1-\frac{R T}{L}
\end{array}\right]\left[\begin{array}{l}
i_{\text {aref }}(k) \\
i_{\beta r e f}(k)
\end{array}\right]+} \\
{\left[\begin{array}{cc}
\frac{T}{L} & 0 \\
0 & \frac{T}{L}
\end{array}\right]\left[\begin{array}{l}
E_{\alpha}-V_{d c}(k) S_{\alpha}(k) \\
E_{\beta}-V_{d c}(k) S_{\beta}(k)
\end{array}\right]}
\end{gathered}
$$

According to (24) we can predict the future value of line current using actual values of line current, source voltage, dc link voltage and switching function.

According to the control objectives, several cost function can be defined, in this work our main objective is current tracking of grid current. So the cost function is selected as:

$$
g(k)=\lambda\left[i_{\text {aref }}(k+1)-i_{\alpha}(k+1)\right]+\gamma\left[i_{\beta r e f}(k+1)-\right.
$$

Where

$\lambda, \gamma$ are the weighting coefficients of line currents.

After sampling the line current and predicting the future values, these values are compared with extrapolated referencing currents. Then the cost function is calculated depending on eight possible cases. The switching state, among the eight possible states, which gives the minimum cost function is chosen and applied to the PWM rectifier gating terminals.

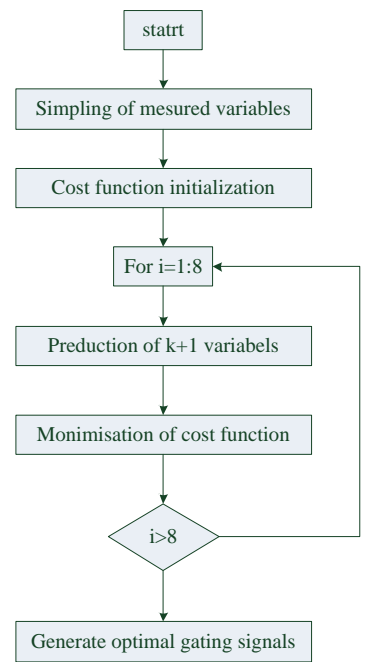

Figure 3. organigram of model predictive control

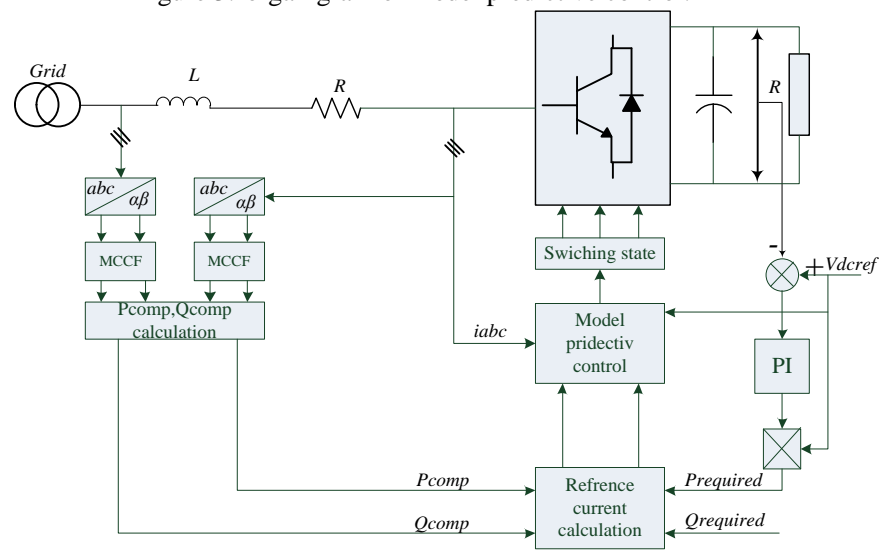

Figure 4. Control diagram of the proposed MPC.

\section{Simulation results}

In order to investigate the effectiveness of the proposed strategy, the control schemes were simulated using MATLAB/ simulink environment, the parameters of the system and the equivalent resistive load are illustrated in tab1.

TABLE I. Power circuit parameters.

\begin{tabular}{|c|c|}
\hline Variable & Value \\
\hline$L_{a}, L_{b}, L_{c}$ & $12 \mathrm{mH}$ \\
\hline$R_{a}, R_{b}, R_{c}$ & $0.3 \Omega$ \\
\hline$C_{d c}$ & $500 \mu \mathrm{F}$ \\
\hline$R$ & $200 \Omega$ \\
\hline Sampling time & $10 \mu \mathrm{s}$ \\
\hline
\end{tabular}

Before checking the performance of the proposed control scheme under unbalanced conditions, we should check the conventional controller for the balanced condition in order to have a comparative benchmark result for the data obtained using a proposed compensating control scheme under unbalanced supply conditions. The beck value of balanced input voltage is $(160 \mathrm{~V})$.

Fig. 5 shows three phase voltage and current, it clearly noted from the wave forms that the line current are sinusoidal and balanced when the source voltage is balance. Also the rectifier work under unity power factor.
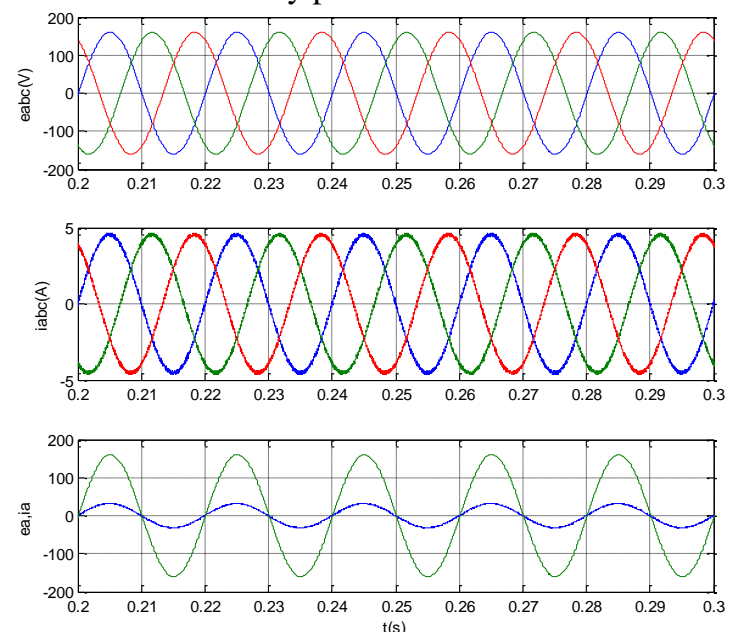
Figure 5. Simulation results from the top to the bottom: 3-phase voltage supply, grid currents, $1^{\text {st }}$ phase voltage and current
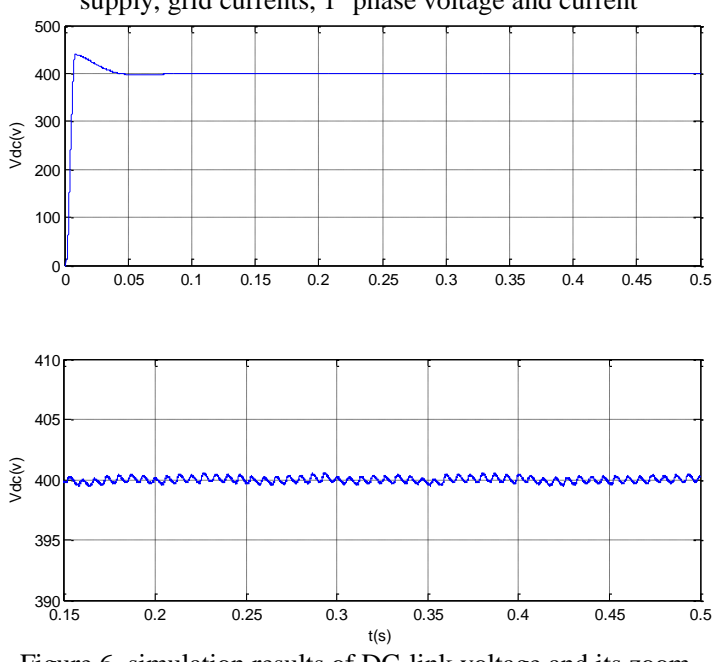

Figure 6. simulation results of DC-link voltage and its zoom.

Fig. 7 shows the dynamic behavior of the 3-phase PWM rectifier when the dc link voltage reference is changed from $400(\mathrm{~V})$ to $450(\mathrm{~V})$ at the instant $0.2 \mathrm{~s}$, then the load value is decreased to $(120 \Omega) 0.3 \mathrm{~s}$.

This figure also demonstrates the robustness of the control scheme via dc link voltage reference variation and it's capable to achieve the reference with small variation and fast transient response time. After decreasing the load the dc link voltage increase slightly and return to its reference with increasing in the current value and the system still stable.

Secondly, the proposed control scheme is tested as the voltage changes from the balance stage to the unbalance one. The unbalanced voltage source in this case is the voltage dips by $15 \%$ in phase a. This type of unbalanced supply is very common in weak AC system where single phase loads are unevenly distributed, or transformers with nonsymmetrical windings are used [3].

Figure 8 shows the dynamic behavior of the PWM rectifier controlled initially by classical MPC under balanced voltages supply and then switched to unbalanced voltages supply at the instant $0.25 \mathrm{~s}$, and then the proposed MPC with the proposed control law was introduced at $0.3 \mathrm{~s}$.
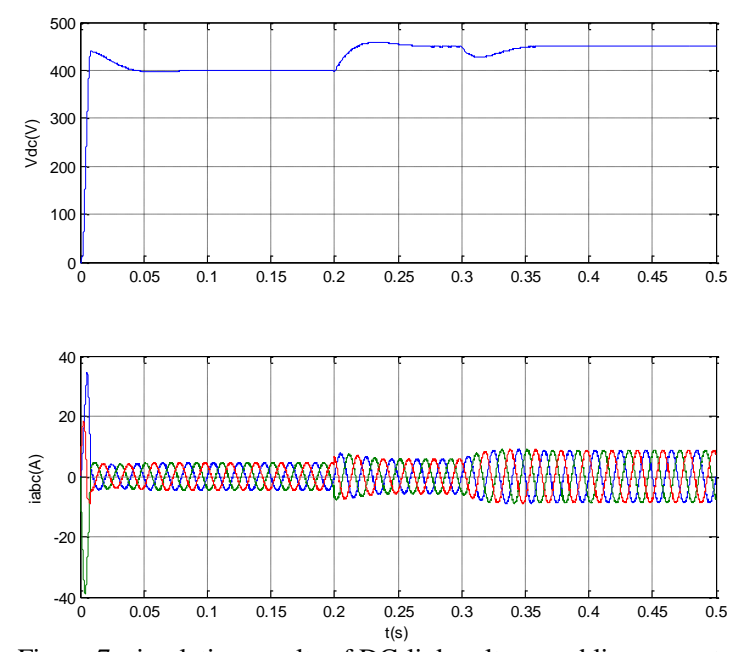

Figure 7. simulation results of DC-link voltage and line current.
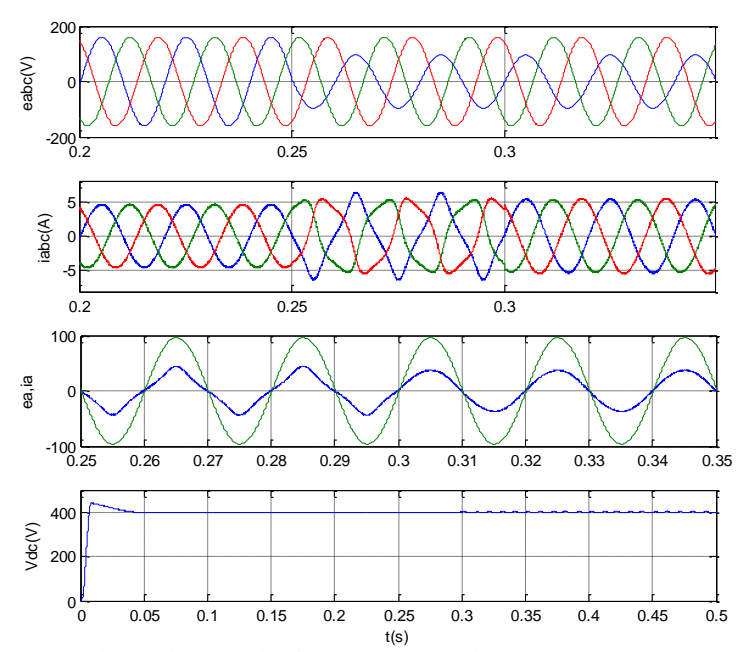

Figure 8. Simulation results from the top to the bottom: 3-phase voltage supply, grid currents, $1^{\text {st }}$ phase voltage and current and DC link voltage.

It can be noted that the classical control strategy offers balanced and sinusoidal grid currents under balanced conditions. But, under unbalanced voltage supply, there are many $3^{\text {rd }}$ order harmonics and unbalanced grid currents. The objective of the improved MPC is to obtain sinusoidal and balanced grid currents under unbalanced voltage supply. It is obvious that after the application of the proposed compensating strategy at time $0.3 \mathrm{~s}$, the low-order harmonics in line currents were eliminated and balanced currents were generated, also unity power factor was obtained under unbalanced grid voltage conditions. Figure 8 shows the DC-link voltage regulation and the voltage balance result for the converter. It is shown that the voltage regulation loop works correctly achieving the voltage reference for each part of the DC-link. This figure also demonstrates the fast transient response time and the robustness of the control scheme under supply variation.

In order to quantify the effectiveness of the proposed control scheme, the harmonic spectrum of input currents has been illustrated in Figure 9. The frequency spectrum for the ideal case with classical control strategy is shown as a benchmark for other cases.

The presence of an unbalance in voltage supply creates pulsation terms in output DC-link voltage, the frequency of the resulting oscillations is twice the input frequency, the reflected pulsations combined with the fundamental of space vector modulation generate the $3^{\text {rd }}$ harmonic input current that is clearly observed in Figure 6.b.

The frequency spectra of these 3 different cases clearly show that the low-order harmonics can be reduced by employing the proposed control under unbalanced voltage supply. The total harmonic distortion (THD) of input currents under ideal supply is $1.92 \%$ and it is increased to $15.88 \%$ under unbalanced supply, and after applying the proposed strategy the THD was decreased to $2.32 \%$. 
Figure a. MCCF structure
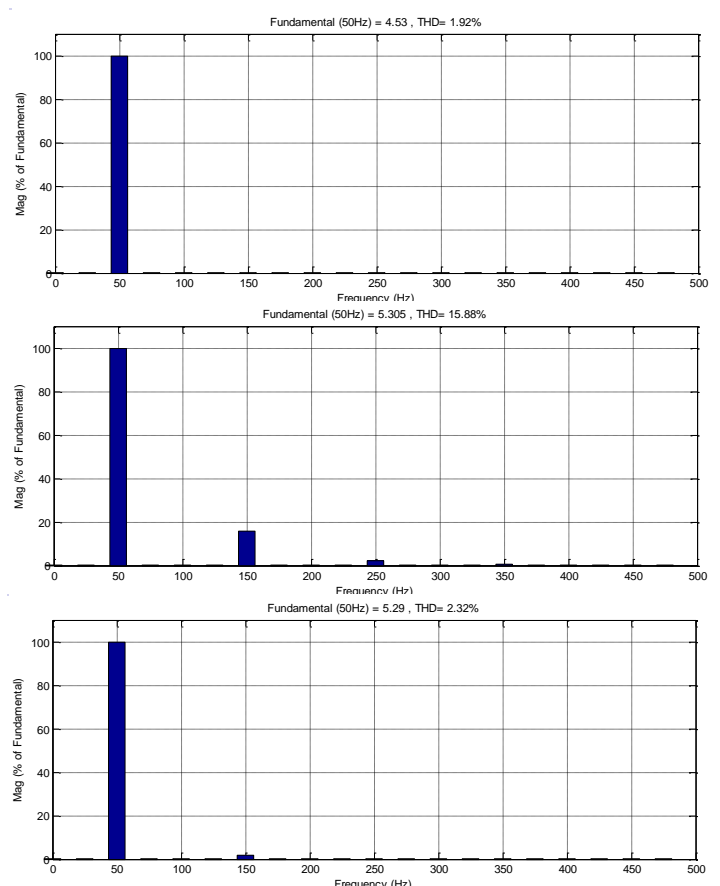

Figure 9. Frequency spectra of input current ia :(a) classical MPC strategy under ideal conditions, (b) classical MPC strategy under unbalanced voltage supply, (c) modified MPC control under unbalanced voltage supply.

\section{Conclusion}

This paper has proposed a modified MPC control strategy for the 3-phase PWM rectifier supplied by unbalanced voltage source. In order to obtain balanced and sinusoidal grid currents under unbalanced voltage conditions, compensated powers are calculated and added to the original referenced power to achieve balanced and high quality input current. The positive, negative and harmonic sequences of the voltage and the current are extracted using MCCF filter. After calculate the referencing current the Model predictive control is introduced to get switching state to apply on semiconductor device. So the proposed control scheme joins the advantage of compensating powers and the fast dynamic response time of MPC. That makes the proposed strategy a strong solution for voltage right through in grid. The modified strategy is verified by simulation under tow cases, which are balanced voltage and unbalanced voltage. And it proves its capability of yielding sinusoidal and balanced grid current with unity power factor under severe non ideal source.

\section{Appendices}

In order to calculate $P$ and $Q$ compensation powers the separation of positive and negative sequences of voltage supply and line currents is needed.

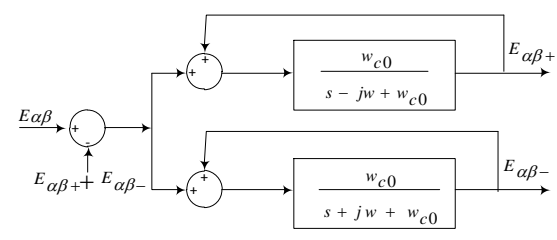

\section{References}

[1] Xiao P, Corzine K.A, Venayagamoorthy G.K. "Multiple reference frame-based control of three-phase PWM boost rectifiers under unbalanced and distorted input conditions" IEEE Trans. Power Electron. 23: 2006-2017.2008.

[2] Moran L, Ziogas P.D, Joos G. Design aspects of synchronous PWM rectifier-inverter systems under unbalanced input voltage conditions. IEEE Trans. Ind. Appl. 28:1286-1293. 1992

[3] Suh Y.S, Lipo T.A. Control scheme in hybrid synchronous stationary frame for PWM AC/DC converter under generalized unbalanced operating conditions. IEEE Trans. Ind. 42: 825-835. Appl. 2006

[4] Etxeberria-outadui I, Viscarret U, Caballero M, Rufer A, Bacha S. New optimized PWM VSC control structures and strategies under unbalanced voltage transients. IEEE Trans. Ind Electron. 54:29022914. 2007

[5] Hu J, Zhang W, Wang H, He Y, Xu L. Proportional and integral plus multi-frequency resonant current controller for grid-connected voltage source converter under imbalanced and distorted supply voltage conditions. J ZHEJIANG UNIV-SCA.10: 1532-1540. 2009

[6] Eloy-Garcia J,Arnaltes S, Redriguez-Amenedo J.L. Direct power control of voltage source inverters with unbalanced grid voltages. IET Power Electron; 1: 395-407. 2007

[7] Shang L, Sun D, Hu J. Sliding-mode-based direct power control of grid connected voltage-sourced inverters under unbalanced network conditions. IET Power Electron. 4: 570-579. 2011

[8] Shang L, Hu J. Sliding-mode-based direct power control of gridconnected wind- turbine-driven doubly fed induction generators under unbalanced grid voltage conditions. IEEE Trans. Energy Conver. 27: 362-373. 2012.

[9] Antoniewicz P. Predictive control of three phase AC/DC converters. PhD Thesis, Warsaw University of Technology, Norway 2009.

[10] ventkata yaramasu, bin wu, Marco rivera and jose Rodriguez. A neaw power conversion system for megawatt PMSG wind turbines using four level converters and simple control scheme based on two-step model predictive strategy-part I: modeling and theoretical analysis. IEEE journal of emerging and selected topics in power electronics,Vol. 2, NO 1, 3-13 MARCH 2014.

[11] Md. Parvez Akter, Saad Mekhilef , Nadia Mei Lin Tan and Hirofumi Akagi. Model Predictive Control of Bidirectional AC-DC Converter for Energy Storage System. J Electr Eng Technol.10(1): 165-175. 2015

[12] Vikas Kumar, Prerna Gaur and Alok Prakash Mitta. Finite-state model redictive control of NPC inverter using multi-criteria fuzzy decision-making. Int. Trans. Electr. Energ. Syst. 25:876-897,2015.

[13] Guo X, Wu W, Chen Z. Multiple-complex coefficient-filter-based phase-locked loop and synchronization technique for three-phase grid-interfaced converters in distributed utility networks. IEEE Trans. Ind. Electron. 58: 1194-1204. 2011

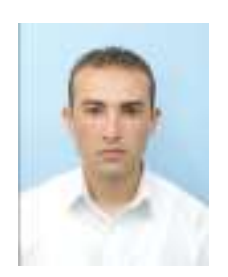

Imad MERZOUK: He Received the Engineering degree in electrical engineering from the University of Jijel, in 2005. And the MS degree from the polytechnics military school (EMP), Algiers, Algeria, in 2008, he is currently preparing a Phd thesis in Batna University, Algeria.

$\mathrm{He}$ is currently an associate professor at department of electrical engineering, university of Djalfa, Algeria. His main fields of interest are control of power converters, power quality, and grid-connected renewable energy. 